\title{
Assessment of fetal heart rate variability and velocity variability by Doppler velocimetry of the descending aorta at 10-20 weeks of gestation
}

\author{
N. T. C. Ursem, E. B. Clark*, B. B. Keller ${ }^{\dagger}$, W. C. J. Hop ${ }^{\ddagger}$ and J. W. Wladimiroff
}

Department of Obstetrics and Gynecology, Academic Hospital Rotterdam-Dijkzigt, Rotterdam, The Netherlands; *Medical Director's Office, University of Utah, School of Medicine, Primary Children's Medical Center, Salt Lake City, Utah, USA; ${ }^{\dagger}$ Department of Pediatrics, University of Kentucky, Lexington, KY, USA; ${ }^{\ddagger}$ Department of Epidemiology and Biostatistics, Erasmus University Rotterdam, Rotterdam, The Netherlands

Key words: DOPPLER ULTRASOUND, DESCENDING AORTA, HEART RATE VARIABILITY, FLOW VELOCITY VARIABILITY

\begin{abstract}
Objectives Determination of gestational age-related modulations in fetal heart rate and descending aorta blood flow velocity in the early human fetus and comparison of aortic variability data with data obtained from the umbilical artery. It is hypothesized that these modulations present in the umbilical artery also occur in the descending aorta.
\end{abstract}

Methods Doppler studies of descending aorta velocity waveforms were performed at 10-20 weeks in 55 normal pregnant women. In 24 of the 55 women, Doppler recordings from both the descending aorta and the umbilical artery were collected. Absolute values and variability of fetal heart rate, peak systolic and time-averaged velocities were determined from flow velocity waveforms of at least $18 \mathrm{~s}$ in duration.

Results From 10 to 20 weeks of gestation, the descending aorta peak systolic and time-averaged velocities increased, whereas the fetal heart rate decreased. The descending aorta peak systolic variability also increased. However, the time-averaged velocity variability and fetal heart rate variability remained constant during the study period. In the subset of 24 women, the fetal heart rate variability and velocity variability data from the descending aorta and umbilical artery were not significantly different.

Conclusions Reproducible fetal heart rate and velocity variability data can be derived from the descending aorta and umbilical artery. The increase in heart rate variability observed in the umbilical artery was not seen in recordings obtained from the descending aorta. Different fetal activity states may be the underlying mechanism for these heart rate variability discrepancies.

\section{INTRODUCTION}

Pulsed Doppler ultrasonography serves as an essential tool for the assessment of human fetal hemodynamics ${ }^{1}$. The late first and early second trimesters of pregnancy are characterized by marked changes in arterial flow velocity waveform patterns, reflecting a marked reduction in fetoplacental vascular resistance ${ }^{2}$. Recently, we have observed that variability in fetal heart rate and umbilical artery flow velocity is gestational age-related in normal pregnancies ${ }^{3}$. Studies from our center have demonstrated altered fetal heart rate variability and umbilical artery flow velocity variability under the pathophysiological conditions of pregnancy-induced hypertension and pregnancies complicated by insulin-dependent diabetes mellitus. Therefore, fetal heart rate variability and flow velocity variability derived from the umbilical artery are likely markers of cardiovascular health in the human fetus at 10-20 weeks of gestation.

Doppler studies from the descending aorta of the chick embryo and fetal lamb have shown that blood flow velocity waveforms are related to fetal cardiac function ${ }^{4,5}$. However, the simultaneous recording of dorsal aortic and peripheral arterial blood velocity is technically challenging $^{6}$. In the human fetus, fetal cardiac output correlates with blood flow in the descending aorta, and aortic peak

Correspondence: Professor J. W. Wladimiroff, Department of Obstetrics and Gynecology, Academic Hospital Rotterdam-Dijkzigt, Dr Molewaterplein 40, 3015 GD Rotterdam, The Netherlands 
systolic velocity may be related to cardiac performance ${ }^{7,8}$. We assumed that regulatory mechanisms, including the autonomic nervous system, function in the combined fetal and umbilical circulations. We therefore hypothesized that the variability observed in the umbilical circulation is also present in the fetal descending aorta.

The objectives of the current study were, first, to determine gestational age-related changes in absolute values for, and beat-to-beat variability in, fetal heart rate, peak systolic velocity and time-averaged velocity derived from descending aorta flow velocity waveforms in normal pregnancies at 10-20 weeks of gestation, and, second, to compare these variables with fetal heart rate and flow velocity data derived from the umbilical artery.

\section{METHODS}

\section{Subjects}

A total of 55 healthy women with a normal singleton pregnancy consented to participate in a cross-sectional study. The study was approved by the Hospital Ethics Committee at the Erasmus University, Rotterdam and the University of Rochester, Rochester, NY. All women were recruited from the outpatient clinic at the University Hospital Rotterdam-Dijkzigt. Gestational age varied between 10 and 20 weeks (median 14 weeks) and was estimated from the last menstrual period and confirmed by ultrasound measurement of the fetal crown-rump length or biparietal diameter. Maternal age ranged between 16 and 43 years (median 28 years). All pregnancies were uncomplicated and resulted in a term delivery of normal infants with a birth weight between the 10th and 90th centiles, corrected for maternal parity and fetal $\operatorname{sex}^{9}$. Women were selected according to five gestational age subgroups (10-11 weeks, $n=12$; $12-13$ weeks, $n=12 ; 14-15$ weeks, $n=12$; 16-17 weeks, $n=10 ; 18-19$ weeks, $n=9$ ) to guarantee a homogeneous distribution. In 24 of the 55 women, Doppler recordings from both the descending aorta and the umbilical artery were collected. The gestational ages of these women had the following distribution: 10-11 weeks, $n=7 ; 12-13$ weeks, $n=6 ; 14-15$ weeks, $n=5 ; 16-17$ weeks, $n=4 ; 18-19$ weeks, $n=2$. The time interval of the Doppler recording between the descending aorta and the umbilical artery was always less than $15 \mathrm{~min}$. Each woman was included in the study only once.

\section{Doppler recordings}

Ultrasound Doppler studies were performed with a Toshiba SSH 140A machine (Toshiba Corp., Medical Systems Division, Tokyo, Japan). A combined transvaginal real-time and color Doppler system (carrier frequency $6 \mathrm{MHz}$ and $5 \mathrm{MHz}$, respectively) was used at 10-13 weeks of gestation and a combined transabdominal real-time and color Doppler system (carrier frequency $5.0 \mathrm{MHz}$ and 3.75 $\mathrm{MHz}$, respectively) was used at 14-20 weeks of gestation. The system operates at power outputs of $<100 \mathrm{~mW} / \mathrm{cm}^{2}$ spatial peak-temporal average in both imaging and
Doppler modes, according to the manufacturer's specification. Doppler recordings were performed by one examiner (NTCU). Flow velocity waveforms from the lower thoracic part of the descending aorta were obtained from a sagittal cross-section through the fetal trunk, displaying a major section of the fetal spine. The vessel interrogation angle was $<30^{\circ}$. Flow velocity waveforms from the umbilical artery were obtained from a straight portion of the freefloating loop of the umbilical cord. The vessel interrogation angle was $<15^{\circ}$. The high-pass filter was set at $100 \mathrm{~Hz}$ and the sample volume length was $0.2-0.3 \mathrm{~cm}$. All Doppler studies were performed with the women in the semirecumbent position and during fetal apnea in the absence of fetal hiccups.

\section{Data processing}

Descending aorta and umbilical artery Doppler recordings were stored on sVHS video tape in PAL format using a Panasonic model AG7350 machine (Matsushita Electric Ind Co., Japan) and digitized at $12 \mathrm{kHz}$ using an AD data acquisition board (LabPC+ and BNC-2081 boards, National Instruments, Austin, TX).

The maximum flow velocity waveforms from the descending aorta and umbilical artery were reconstructed from the Doppler audio data using computer algorithms developed in our center using LabVIEW software (National Instruments, Austin, TX) (Figure 1). Digitized
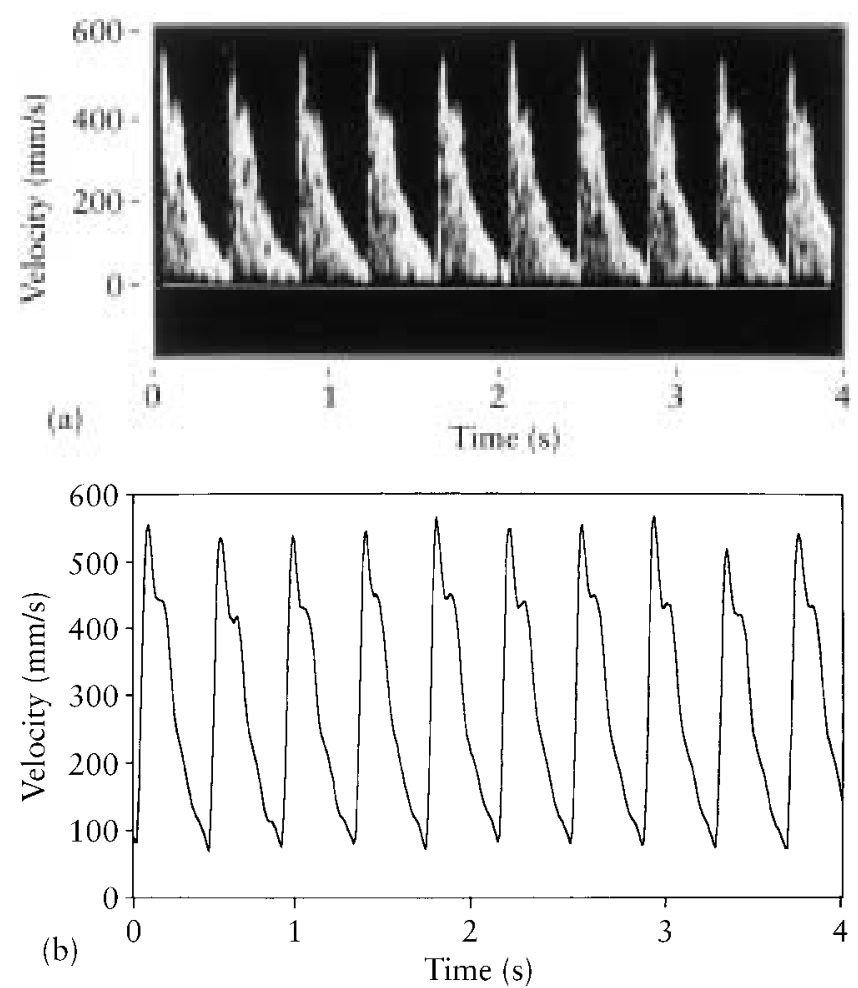

Figure 1 (a) Doppler ultrasound recording of blood flow velocity waveforms from the thoracic part of the descending aorta at 18 weeks of gestation. (b) Schematic presentation of the estimated maximum velocity envelope of the descending aorta at 18 weeks of gestation; this is used for calculation of heart rate and velocity variability data 
audio data packets of 512 points and $75 \%$ overlap were used with Hanning windowing prior to Fourier transform spectral analysis. From the collected power spectra the maximum frequency (velocity) can be estimated. A detailed description of the analysis technique and the computer algorithms has been published previously ${ }^{10}$. For the entire maximum flow velocity waveform, the peak systolic velocity, time-averaged velocity and heart rate per cardiac cycle were calculated. The standard deviation (SD) over a period of at least 45 consecutive heart beats was used as a measure of the fetal heart rate variability and the coefficient of variation $(\mathrm{CV})$ over a period of at least 45 consecutive heart beats was used as a measure of velocity variability. The duration of the Doppler recording of the descending aorta and umbilical artery ranged between 18 and $44 \mathrm{~s}$ (median 24 s), including 44-118 (median 65) high-quality waveforms.

\section{Reproducibility study}

A previous reproducibility study of the umbilical artery flow velocity waveform recordings at 10-20 weeks of gestation revealed a mean $\mathrm{CV}$ of $1.1 \%$ (range $0.2-3.5 \%$ ) for fetal heart rate, a mean CV of $1.9 \%$ (range $1.1-3.3 \%$ ) for peak systolic velocity and a mean CV of $2.4 \%$ (range $1.2-3.9 \%$ ) for time-averaged velocity ${ }^{11}$.

The reproducibility of the descending aorta flow velocity waveform recordings was established in a separate study of 11 singleton pregnancies at 10-20 weeks of gestation. In each of the 11 fetuses three independent measures were made at 5-min intervals. For each of the waveform parameters (fetal heart rate, peak systolic velocity and timeaveraged velocity) the mean $\mathrm{CV}$ was determined.

\section{Statistical analysis}

For each fetus, the mean and SD were calculated for heart rate, absolute peak systolic velocity and absolute time-averaged velocity, as well as fetal heart rate variability. For the expression of variability in the velocity parameters we used the $\mathrm{CV}$, because the SD of peak systolic and time-averaged velocities was not independent of the mean. A logarithmic transformation was performed for the absolute velocity parameters, velocity variability and heart rate variability to stabilize the variance for gestational age. For all six variables, i.e. mean and SD of heart rate, mean and $\mathrm{CV}$ of peak systolic and time-averaged velocities, linear regression was used to evaluate the relationship between these variables and gestational age. The 50th, 10th and 90th centiles were established using the mean and the mean \pm 1.64 SD of the residuals. A value of $p \leq 0.05$ was taken as the limit of significance. For comparison of the velocity and heart rate parameters determined from the descending aorta and umbilical artery, a paired Wilcoxon's test was used. All calculations were performed with SPSS 6.1 software (SPSS Inc., Chicago, IL, USA).

\section{RESULTS}

The reproducibility study of the descending aorta revealed a mean $\mathrm{CV}$ of $0.7 \%$ (range $0.1-2.3 \%$ ) for fetal heart rate; $2.2 \%$ (range $0.3-4.4 \%$ ) for peak systolic velocity and $3.0 \%$ (range $0.2-5.8 \%$ ) for time-averaged velocity.

During the gestational age period of 10-20 weeks, the descending aorta peak systolic velocity (slope $=0.04$, $r=0.44, p<0.001$ ) and time-averaged velocity (slope $=$ $0.07, r=0.60, p<0.001)$ increased, whereas the instantaneous fetal heart rate decreased (slope $=-2.52, r=0.71$, $p<0.001)$. A statistically significant increase was demonstrated for the descending aorta peak systolic velocity variability at 10-20 weeks (Figure 2). The descending aorta time-averaged velocity variability (Figure 3 ) and fetal heart rate variability (Figure 4) remained constant during the entire study period.

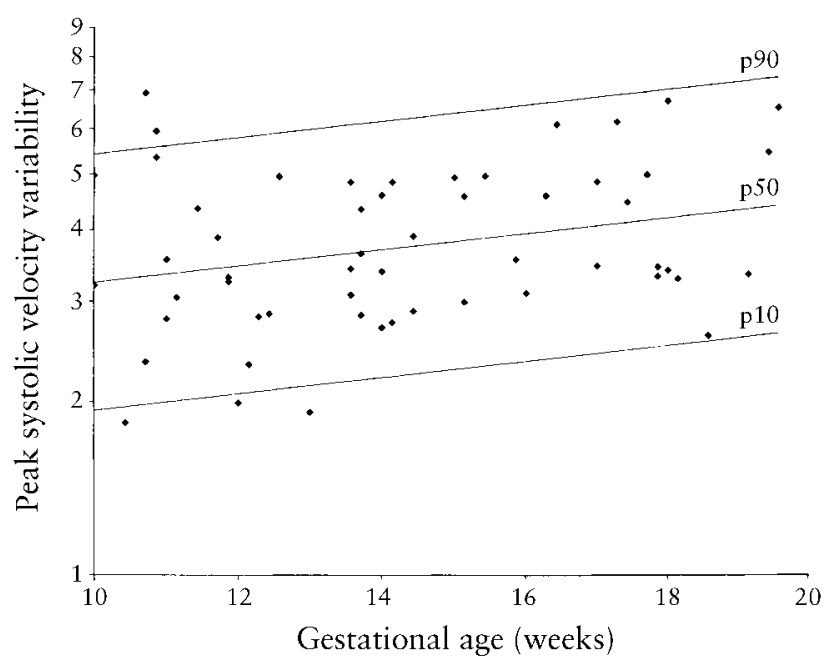

Figure 2 Individual data and centiles (10th, 50th and 90th) for descending aorta peak systolic velocity variability $(\%)$ expressed as $\mathrm{CV}$ relative to gestational age. Note the logarithmically transformed vertical axis (slope $=0.03, r=0.27, p=0.04$ )

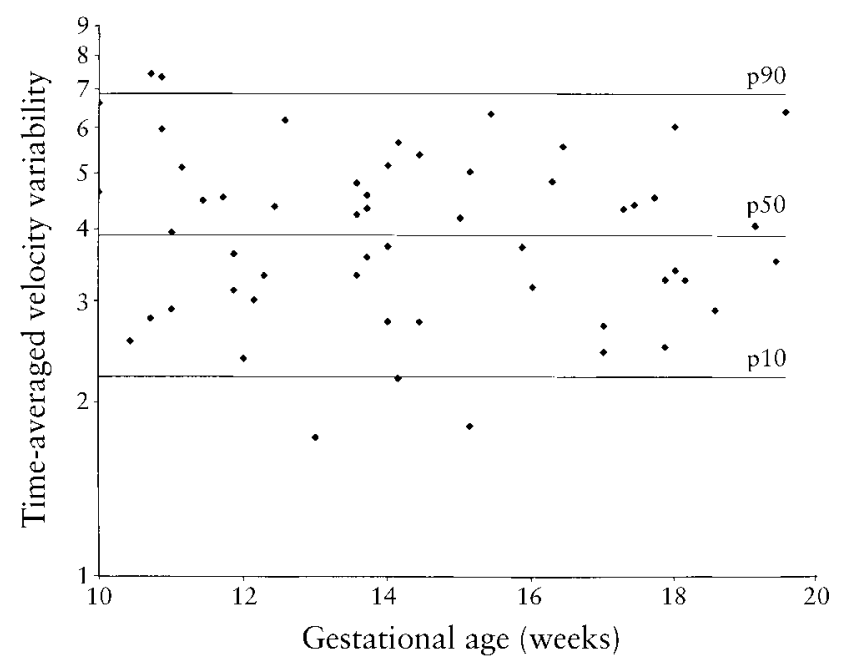

Figure 3 Individual data and centiles (10th, 50th and 90th) for descending aorta time-averaged velocity variability (\%) expressed as CV relative to gestational age. Note the logarithmically transformed vertical axis 
Comparison of the velocity and fetal heart rate parameters between the descending aorta and umbilical artery in the subset of 24 women is displayed in Table 1 . The peak systolic and time-averaged velocities were significantly higher in the descending aorta than in the umbilical artery. These differences were not gestational age-dependent. The remaining parameters were not significantly different between the two vessels.

\section{DISCUSSION}

The aim of this study was to determine the gestational age-related changes in absolute values for, and beat-to-beat variability in, fetal heart rate, peak systolic velocity and time-averaged velocity derived from descending aorta flow velocity waveforms in normal pregnancies at 10-20 weeks of gestation. The aortic time-averaged velocity multiplied by the cross-sectional area equals the aortic blood flow. Blood flow in the descending aorta represents $70-75 \%$ of cardiac output in both the human and the sheep fetus in late gestation ${ }^{4}$ and $90 \%$ of cardiac output in the chick embryo $^{6,12}$. Therefore, aortic blood flow can be considered an index of cardiac output during the growth and morphogenesis of the cardiovascular system. The gestational agerelated rise in descending aortic peak systolic velocity observed in the present study may be accounted for by a

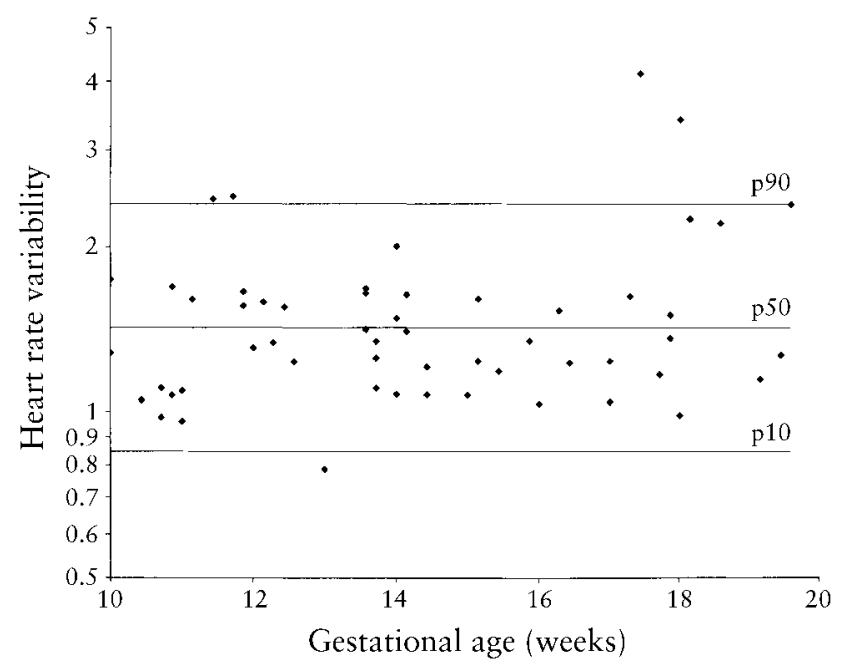

Figure 4 Individual data and centiles (10th, 50th and 90th) for heart rate variability (beats/min) expressed as SD relative to gestational age. Note the logarithmically transformed vertical axis fetal growth-determined increase in volume flow, a reduction in afterload or increased myocardial contractility. Measurements of cardiac function in 18- and 28-day-old rabbits showed marked developmental changes in the myocardial contractile system, producing a more efficient contraction pattern ${ }^{13}$. The gestational age period studied in the rabbit (18-20 days) is comparable to 10-18 weeks of gestation in the human fetus ${ }^{14}$. Thus, improvement in myocardial contractility may be partly responsible for the increased fetal descending aortic flow velocities we observed. The non-invasive nature of the fetal Doppler studies does not allow differentiation between variables such as volume flow, afterload and contractility. We can, therefore, only speculate that the gestational age-related increase in descending aortic peak flow velocity may have been due to one or a combination of these variables.

As expected, absolute peak systolic and time-averaged velocities in the descending aorta were higher than in the umbilical artery (Table 1), since absolute velocities decrease from the central to the distal level.

Doppler recordings of the descending aorta consisted of 18-44 s of data, ensuring a minimum of 44 cardiac cycles for analysis of heart rate and velocity variability. Fetal heart rate and time-averaged velocity variability determined from the descending aorta remained similar during the study period of 10-20 weeks of gestation, whereas peak systolic velocity variability increased significantly with advancing gestation. Peak systolic velocity is more susceptible to subtle maternal and/or fetal movements than time-averaged velocity. Movements such as maternal breathing may shift the position of the interrogation angle or the Doppler sample volume, so that different velocities will be recorded. However, a previous study from our center demonstrated that spontaneous maternal breathing does not influence heart rate and peak systolic velocity variability data measured in the umbilical artery.

A recent cross-sectional Doppler flow velocity study in the umbilical artery ${ }^{11}$ demonstrated an increase in umbilical artery peak systolic velocity variability at 10-20 weeks and an increase in fetal heart rate variability at 15-20 weeks. We speculated that the increased heart rate variability was mediated by maturation of the parasympathetic nervous system, whereas the rise in peak systolic velocity variability reflected the activation of a hemodynamic feedback mechanism, similar to that demonstrated in the chick embryo $^{15}$.

Table 1 Median and range for fetal heart rate, peak systolic velocity, time-averaged velocity, heart rate variability, peak velocity variability and time-averaged velocity variability determined in both the descending aorta and the umbilical artery of each fetus $(n=24)$

\begin{tabular}{|c|c|c|c|c|}
\hline & \multicolumn{2}{|c|}{ Descending aorta } & \multicolumn{2}{|c|}{ Umbilical artery } \\
\hline & Median & Range & Median & Range \\
\hline Peak systolic velocity $(\mathrm{mm} / \mathrm{s})$ & $328.8 *$ & $211.3-765.4$ & 275.3 & $156.5-399.9$ \\
\hline Time-averaged velocity $(\mathrm{mm} / \mathrm{s})$ & $153.2 *$ & $88.1-368.6$ & 125.9 & $59.3-227.5$ \\
\hline Heart rate (beats/min) & 154 & $145-187$ & 154 & $146-187$ \\
\hline Peak systolic velocity variability (CV) & 3.40 & $1.84-6.54$ & 3.09 & $1.65-7.20$ \\
\hline Time-averaged velocity variability (CV) & 4.10 & $1.74-6.42$ & 3.57 & $1.57-8.08$ \\
\hline Heart rate variability $(\mathrm{SD})$ & 1.34 & $0.79-4.13$ & 1.48 & $1.05-2.29$ \\
\hline
\end{tabular}

$\mathrm{CV}$, coefficient of variation; SD, standard deviation; *, $p<0.05$ by paired Wilcoxon's test 
In this study, fetal heart rate variability derived from the descending aorta remained unchanged throughout the study period. A possible explanation for the difference in heart rate variability derived from the descending aorta and umbilical artery might be the nature of the fetal activity state at the time of the Doppler survey. The recording conditions might not change in the umbilical artery despite minor fetal movements, whereas assessment of flow velocity waveforms in the fetal descending aorta is feasible only when the fetus is lying completely still during the recording session. In normally developing fetuses, coupling between fetal movement and fetal heart rate has been established as early as 20 weeks of gestation ${ }^{16}$. Behavior patterns can be observed in the human fetus even before 20 weeks of gestation $^{17,18}$. Quiescence, active movements involving all parts of the body, isolated limb movements and strong pulsed trunk movements can be observed in fetuses between 10 and 12 weeks of gestation, and these closely resemble behavioral patterns established later in pregnancy ${ }^{18}$. We propose that a low fetal activity state is required to obtain technically acceptable waveforms from the descending aorta, whereas waveform recordings of the umbilical artery may be technically acceptable under conditions that represent both low and high activity states; the latter is associated with (minor) fetal movements.

We conclude from this study that reproducible fetal heart rate and velocity variability data can be derived from the descending aorta. However, fetal heart rate variability data established from the descending aorta differ from the variability data obtained from the umbilical artery. Different fetal activity states may be the underlying mechanism for these heart rate variability discrepancies.

\section{ACKNOWLEDGEMENTS}

This study was supported by National Institute of Health Grant P50-HL51498, SCOR in Pediatric Cardiovascular Disease at the University of Rochester, Rochester, NY.

\section{REFERENCES}

1. Wladimiroff JW, Huisman TW, Stewart PA. Intracerebral, aortic, and umbilical artery flow velocity waveforms in the late-first-trimester fetus. Am J Obstet Gynecol 1992;166:46-9
2. Wladimiroff JW, Huisman TW, Stewart PA. Fetal cardiac flow velocities in the late 1st trimester of pregnancy: a transvaginal Doppler study. J Am Coll Cardiol 1991;17:1357-9

3. Ursem NTC, Kempski MH, Ridder dMAJ, Clark EB, Wladimiroff JW. An estimate of fetal autonomic state by spectral analysis of human umbilical artery flow velocity waveforms. Cardiovasc Res 1998;37:601-5

4. Thompson RS, Trudinger BJ, Reed VD, Turner AJ. Aortic Doppler velocity measurement and cardiac function in the fetal lamb. Ultrasound Med Biol 1994;20:893-902

5. Clark EB, Hu N. Developmental hemodynamic changes in the chick embryo from stage 18 to 27. Circ Res 1982;51:810-15

6. Yoshigi M, Ettel JM, Keller BB. Developmental changes in flow-wave propagation velocity in embryonic chick vascular system. Am J Physiol 1997;273:H1523-9

7. Tonge HM, Wladimiroff JW, Noordam MJ, van Kooten C. Blood flow velocity waveforms in the descending fetal aorta: comparison between normal and growth-retarded pregnancies. Obstet Gynecol 1986;67:851-5

8. Wladimiroff JW, McGhie J. Ultrasonic assessment of cardiovascular geometry and function in the human fetus. $\mathrm{Br} J$ Obstet Gynaecol 1981;88:870-5

9. Kloosterman G. On intrauterine growth. Int J Obstet Gynaecol 1970;8:895-912

10. Ursem NTC, Brinkman HJF, Struijk PC, Hop WCJ, Kempski $\mathrm{MH}$, Keller BB, Wladimiroff JW. Umbilical artery waveform analysis based on maximum, mean and mode velocity in early human pregnancy. Ultrasound Med Biol 1998;24:1-7

11. Ursem NTC, Struijk PC, Hop WCJ, Clark EB, Keller BB, Wladimiroff JW. Heart rate and flow velocity variability as determined from umbilical Doppler velocimetry at 10-20 weeks of gestation. Clin Sci 1998;95:539-45

12. Hu N, Clark EB. Hemodynamics of the stage 12 to stage 29 chick embryo. Circ Res 1989;65:1665-70

13. Nakanishi T, Seguchi M, Takao A. Development of the myocardial contractile system. Experientia 1988;44:936-44

14. Sissman NJ. Developmental landmarks in cardiac morphogenesis: comparative chronology. Am J Cardiol 1970;25: $141-8$

15. Kempski MH, Kibler N, Blackburn JL, Dzakowic J, Hu N, Clark EB. Hemodynamic regulation in the chick embryo. ASME Adv Bioeng 1993;24:119-22

16. Dipietro JA, Hodgson DM, Costigan KA, Hilton SC, Johnson TR. Development of fetal movement - fetal heart rate coupling from 20 weeks through term. Early Hum Dev 1996;44:139-51

17. De Vries JI, Visser GH, Prechtl HF. Fetal behaviour in early pregnancy. Eur J Obstet Gynecol Reprod Biol 1986;21:271-6

18. Van Dongen LG, Goudie EG. Fetal movement patterns in the first trimester of pregnancy. Br J Obstet Gynaecol 1980;87: 191-3 